\title{
Prevalence of hyperuricemia among the Chinese population of the southeast coastal region and association with single nucleotide polymorphisms in urate-anion exchanger genes: SLC22A12, ABCG2 and SLC2A9
}

\author{
XINRAN YANG $^{1 *}$, YINGXIU XIAO $^{2 *}$, KAIXI LIU $^{3}$, XIAOYANG JIAO $^{4}$, \\ XIAOZHE LIN ${ }^{1}$, YONGNI WANG ${ }^{1}$ and QIAOXIN ZHANG ${ }^{1}$

\begin{abstract}
Departments of ${ }^{1}$ Clinical Laboratory and ${ }^{2}$ Neurology, The First Affiliated Hospital of Shantou University Medical College;
${ }^{3}$ Department of Clinical Laboratory, Shantou Central Hospital, Shantou, Guangdong 515041;

${ }^{4}$ Medical College of Shantou University, Shantou, Guangdong 515043, P.R. China
\end{abstract}

Received December 27, 2017; Accepted May 15, 2018

DOI: $10.3892 / \mathrm{mmr} .2018 .9290$

\begin{abstract}
Genome-wide association studies identified that a series of genes, including solute carrier family (SLC) 2 member 9 (SLC2A9), SLC 22 member 12 (SLC22A12) and ATP-binding cassette sub-family G member 2 (ABCG2) polymorphisms were associated with serum uric acid (SUA) levels in the present study. High incidence rates of hyperuricemia were reported in the Chinese population of the southeast coastal region; however, no evidence has confirmed the genetic association with SUA levels in this region. The present study aimed to investigate the association between uric acid levels and hyperuricemia, and genotypes of the Chinese population of the southeast coastal region. In the present study, a total of 1,056 healthy patients attending routine checkups were employed to investigate the incidence of hyperuricemia; 300 subjects were then randomly selected from the 1,056 patients for the identification of genetic polymorphisms of SLC2A9rs11722228, SLC22A12rs893006 and ABCG2rs2231142 via high-resolution melting. The present study reported that the incidence rate of hyperuricemia was $32.6 \%$ (42.5\% in males and $22.7 \%$ in females, respectively). The prevalence of ABCG2rs2231142 polymorphisms (CC, CA and AA) was $44.4,44.8$ and $11.8 \%$, respectively;
\end{abstract}

Correspondence to: Dr Qiaoxin Zhang, Department of Clinical Laboratory, The First Affiliated Hospital of Shantou University Medical College, 57 Changping Road, Shantou, Guangdong 515041, P.R. China

E-mail: zhangqx2015@126.com

${ }^{*}$ Contributed equally

Abbreviations: SNP, single nucleotide polymorphism; SUA, serum uric acid; HRM, high-resolution melting; GWAS, genome-wide association studies; OR, odds ratio; $\mathrm{CI}$, confidence interval

Key words: hyperuricemia, polymorphism, high-resolution melting
SLC2A9rs11722228 polymorphisms (CC, CT and TT) were reported to be $49.3,40.3$ and $10.3 \%$, respectively. Additionally, SLC22A12rs893006 polymorphisms (CC, CT and TT) were determined to be $57.2,38.7$ and $4.1 \%$, respectively. The SUA levels were observed to be statistically different among each investigated genotype of ABCG2rs2231142 ( $\mathrm{P}=0.047)$. The A allele was significantly associated with an increased risk of hyperuricemia (odds ratio=2.405 and 1.133 for CA and AA, respectively). The present study reported that high incidence rates of hyperuricemia in the Chinese population of the southeast coastal region may be closely associated with the variants of ABCG2rs2231142. Whether polymorphisms of SLC2A9rs11722228 and SLC22A12rs893006 are involved in hyperuricemia require further investigation.

\section{Introduction}

Uric acid (UA) is the final metabolic product of purine metabolism in humans (1). A total of two-thirds of the uric acid within the human body is normally excreted via the kidneys; the remaining third is excreted via the intestinal tract (1). Elevated serum uric acid (SUA) levels have been strongly associated with myocardial syndrome, diabetes and cardiovascular disease, and an increased risk of mortality in mature individuals ( $\geq 18$ years old) $(2)$.

SUA levels are governed by the balance between purine absorbed from the diet and that excreted via the kidneys. In $>90 \%$ of cases, elevated SUA levels result from impaired renal excretion $(1,3,4)$. Genetic factors may affect the critical reabsorption process of UA in the proximal renal tubule, which may contribute to $40-70 \%$ of the overall SUA levels $(5,6)$. Previous genome-wide association studies (GWAS) have identified an association between multiple gene loci, including solute carrier family (SLC) 2 member 9 (SLC2A9)/glucose transporter 9 (GLUT9), ATP-binding cassette sub-family G member 2 (ABCG2), SLC 22 member 12 [SLC22A12/urate transporter 1 (URAT1)], SLC 22 member 11 and SLC 16 member 9 and SUA levels in the European population $(4,7)$. It 
has been previously indicated that SUA levels are influenced by gene-environment interactions (8). Studies have investigated the association between SUA levels and genotype in Japanese and Korean populations (9-11); however, further investigation of the Chinese population is required (12).

It has been reported that the Chinese population of the southeast coastal region have a diet rich in seafood, which may be associated with the onset of hyperuricemia (13). However, strict control of diet alone is insufficient to reduce the levels of SUA within this region $(14,15)$, indicating that dysregulation of UA excretion may be a principal factor associated with hyperuricemia; polymorphisms in urate-anion exchanger genes may serve an important role in this process. At present, no epidemiological investigations into the association between hyperuricemia and genotype have been performed in this region. The present study aimed to investigate the epidemiology of hyperuricemia in the Chinese population of the southeast coastal region; a total of three single nucleotide polymorphism (SNPs) were selected (SLC2A9rs11722228, SLC22A12rs893006 and ABCG2rs2231142) to examine their associations with SUA levels.

High-resolution melting (HRM) is a novel molecular approach that was originally developed for SNP genotyping and detection (16). This method employs a single-step closed tube, facilitating an economical procedure for rapid, specific and sensitive detection. In the present study, an HRM assay was generated to detect three functional SNPs; the genetic association of these SNPs with SUA levels was also examined.

\section{Materials and methods}

Subjects and data collection. A total of 1,056 healthy patients attending routine checkups (543 males and 514 females; aged 20-50 years old) at the Medical Examination Center of The First Affiliated Hospital of Shantou University Medical College (Shantou, China) were enrolled in the present study between January 2015 and December 2015. The inclusion criteria were as follows: Serum creatinine levels $\leq 133 \mu \mathrm{mol} / 1$ for males and $\leq 108 \mu \mathrm{mol} / 1$ for females. Hyperuricemia was defined as SUA levels exceeding $420 \mu \mathrm{mol} / \mathrm{l}$ in males and $360 \mu \mathrm{mol} / 1$ in females. The characteristics of participants, including age, sex, body mass index, total cholesterol, triglyceride, low-density lipoprotein, high-density lipoprotein, glucose levels and place of origin are reported (Table I). A total of 300 subjects were randomly selected from the 1,056 patients for polymerase chain reaction (PCR)-HRM genotyping analysis. All participants provided written informed consent for enrolment into the present study and for genotype analysis. The present study was approved by the Ethics Committee of The First Affiliated Hospital of Shantou University Medical College.

Measurement of SUA levels. Venous blood samples (3 ml) were drawn from the individuals following an overnight fast. SUA levels were measured using a uricase-peroxidase method (17) via an automated system (Beckman AU5800; Beckman Coulter, Inc., Brea, CA, USA).

PCR-HRM. Genomic DNA was extracted from venous blood samples $(3 \mathrm{ml})$ using a Lab-Aid ${ }^{\circledR} 824$ DNA Extraction kit (Xiamen Zeesan Biotech, Co., Ltd., Xiamen China) according to the manufacturer's protocol. Oligonucleotide primers were designed using primer premier5 software (http://macdownload.informer.com/advice/Primer_Premier_5. html) and synthesized by Sangon Biotech Co., Ltd. (Shanghai, China) (Table II). Each PCR employed 30 ng DNA, 2X HRM PCR Master Mix (Qiagen, Inc., Valencia, CA, USA) and $10 \mu \mathrm{M}$ primer mix; the solution was made up to $25 \mu \mathrm{l}$ with RNase-Free water. PCR was performed on a LightCycler 480 system (Roche Diagnostics, Basel, Switzerland). All reactions were conducted under the following conditions: $95^{\circ} \mathrm{C}$ for $5 \mathrm{~min}$ (1 cycle), 35 cycles of $95^{\circ} \mathrm{C}$ for $10 \mathrm{sec}$ and $55^{\circ} \mathrm{C}$ for $30 \mathrm{sec}$. The optimum annealing temperature and amplified fragment size for each SNP was modified according to the primer design (Table II).

Following PCR, the amplified fragments were confirmed by electrophoresis using $1.5 \%(\mathrm{w} / \mathrm{v})$ agarose gels, which were subsequently visualized using $0.5 \mu \mathrm{g} / \mathrm{ml}$ ethidium bromide. Following this, a total of 50 DNA samples were randomly selected from 300 samples for genotype analysis by sequencing (as described below). Analysis of the wild-type, heterozygous and homozygous type for each SNP was conducted, which was compared with the 50 DNA samples of known genotype represented the control in the following HRM analysis steps.

DNA samples as mentioned previously as well as the unknown samples were examined via PCR-HRM curves mixed with known genotype samples (wild-type, heterozygous and homozygous). PCR thermocycling and HRM were performed on a LightCycler 480 (Roche Diagnostics). All reactions were detected under the following conditions: $95^{\circ} \mathrm{C}$ for $5 \mathrm{~min}$ ( 1 cycle), 35 cycles of $95^{\circ} \mathrm{C}$ for $10 \mathrm{sec}$ and $55^{\circ} \mathrm{C}$ for $30 \mathrm{sec}$, and a melting stage from $65-95^{\circ} \mathrm{C}$, which was reported at intervals of $0.02^{\circ} \mathrm{C} / \mathrm{sec}$. HRM curves were analyzed using Roche 4802.0 software (Roche Diagnostics).

Sequencing. To further verify the results of PCR-HRM genotyping, 50 DNA samples were randomly selected from the total 300 samples and examined via sequencing. Novel primers (Table II) were designed for the sequencing of gene regions containing SNPs that yielded products with lengths $>200$ bp. DNA sequencing was performed by Sangon Biotech Co., Ltd.

Bioinformatics analysis. Linkage disequilibrium analyses were performed using Haploview 4.2 software (www.broadinstitute.org/haploview/downloads). $\mathrm{R}^{2}>0.8$ was considered to represent complete linkage disequilibrium. DNAMAN (version 8.0; Lynnon BioSoft, Vaudreuil, QC, Canada) was used for DNA sequence alignment between the National Center for Biotechnology Information database (http://www. ncbi.nlm.nih.gov) and the sequencing results generated by the present study.

Statistical analysis. The Hardy-Weinberg equilibrium of the three SNPs was examined with a $\chi^{2}$ test. Analysis of variance was used to evaluate the difference in SUA levels in each genotype followed by the Least Significant Difference post-hoc test. The odds ratio (OR) and 95\% confidence interval (CI) were calculated by a binary logistic model to identify factors that may influence the concentration of SUA. All statistical analyses were performed using SPSS 20.0 
Table I. Characteristics of patients.

\begin{tabular}{lcccc}
\hline & \multicolumn{2}{c}{ Hyperuricemia $^{\mathrm{a}}$} & \multicolumn{2}{c}{ Normal serum uric acid } \\
\cline { 2 - 3 } Characteristics & Male & Female & Male \\
\hline No. of patients (\%) & $231(42.5)$ & $117(22.7)$ & $312(57.5)$ & $397(77.3)$ \\
Age, years & $37.02 \pm 12.56$ & $37.01 \pm 12.86$ & $37.11 \pm 11.79$ & $35.51 \pm 14.58$ \\
Body mass index & $21.77 \pm 0.96$ & $22.01 \pm 1.91$ & $20.73 \pm 1.23$ & $21.62 \pm 1.33$ \\
Creatinine & $110.95 \pm 15.96$ & $95.33 \pm 9.86$ & $109.06 \pm 18.91$ & $92.36 \pm 14.21$ \\
Triglycerides & $3.02 \pm 1.16$ & $1.68 \pm 0.91$ & $1.99 \pm 0.96$ & $1.59 \pm 0.54$ \\
Total cholesterol & $7.33 \pm 3.82$ & $5.92 \pm 1.27$ & $5.32 \pm 3.61$ & $4.96 \pm 2.66$ \\
Low density lipoprotein & $3.57 \pm 2.21$ & $2.97 \pm 3.64$ & $2.21 \pm 1.09$ & $1.98 \pm 0.61$ \\
High density lipoprotein & $1.21 \pm 0.33$ & $1.11 \pm 0.51$ & $1.19 \pm 0.51$ & $7.1 \pm 0.32$ \\
Glucose & $7.56 \pm 2.21$ & $7.02 \pm 3.31$ & $3.11 \pm 2.17$ \\
Serum uric acid, $\mu$ mol/1 & 494.01 & 421.24 & 356.94 \\
\hline
\end{tabular}

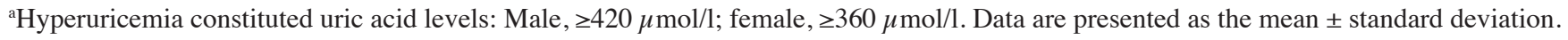

Table II. Primer sequences.

\section{A, PCR-HRM}

\begin{tabular}{|c|c|c|c|c|}
\hline SNP & SNP Allele & Sequence $\left(5^{\prime} \rightarrow 3^{\prime}\right)$ & Size, bp & Temperature \\
\hline SLC2A9 rs11722228 & $\mathrm{C} / \mathrm{T}$ & $\begin{array}{l}\text { F: TGAGTGGCAGAGCTGAGATCG } \\
\text { R: TCTCACCCAGAGGTAGCAGAG }\end{array}$ & 73 & $55^{\circ} \mathrm{C}$ \\
\hline SLC22A12 rs893006 & $\mathrm{C} / \mathrm{A}$ & $\begin{array}{l}\text { F: CCACAATCCCTAGGAGGGAGA } \\
\text { R: TGCTACCCTGTACCCACTGC }\end{array}$ & 146 & $60^{\circ} \mathrm{C}$ \\
\hline ABCG2 rs 2231142 & $\mathrm{C} / \mathrm{A}$ & $\begin{array}{l}\text { F: TGTCTCATTAAAATGCTATTTGCCT } \\
\text { R: GTTGCAAGCCGAAGAGCTG }\end{array}$ & 100 & $61^{\circ} \mathrm{C}$ \\
\hline
\end{tabular}

B, Genotyping

\begin{tabular}{|c|c|c|c|c|}
\hline SNP & SNP Allele & Sequence $\left(5^{\prime} \rightarrow 3^{\prime}\right)$ & Size, bp & Temperature \\
\hline SLC2A9 rs11722228 & $\mathrm{C} / \mathrm{T}$ & $\begin{array}{l}\text { F: TGTGGCTGGGTCTAGCATCT } \\
\text { R: ATTTGCTTGGGGCTGTGGAT }\end{array}$ & 623 & $59^{\circ} \mathrm{C}$ \\
\hline SLC22A12 rs893006 & $\mathrm{C} / \mathrm{A}$ & $\begin{array}{l}\text { F: GACAGGAAGGCCATGTGGTAT } \\
\text { R: CTCCTCCTCTCTGAGACCTTCT }\end{array}$ & 713 & $60^{\circ} \mathrm{C}$ \\
\hline ABCG2 rs2231142 & $\mathrm{C} / \mathrm{A}$ & $\begin{array}{l}\text { F: GGCCCTTGTAAGGACAGTGAT } \\
\text { R: CTCСTCСТCTCTGAGACCTTCT }\end{array}$ & 391 & $59^{\circ} \mathrm{C}$ \\
\hline
\end{tabular}

F, forward primer; R, reverse primer; SNP, single nucleotide polymorphism.

(IBM Corp., Armonk, NY, USA). P<0.05 was considered to indicate a statistically significant difference.

\section{Results}

Epidemiological analysis. The epidemiological investigation of the Chinese population in the southeast coastal region revealed that the mean levels of SUA were $365.27 \pm 116.85 \mu \mathrm{mol} / 1$ $(410.25 \pm 110.36 \mu \mathrm{mol} / 1$ for males; $320.39 \pm 102.12 \mu \mathrm{mol} / 1$ for females; data not shown). Based on the diagnostic criteria, a mean of $32.6 \%$ subjects were considered to have hyperuricemia ( $42.5 \%$ for males; $22.7 \%$ for females). The characteristics of patients are presented in Table I.

Distribution of the three SNP genotypes. In the present study, SLC2A9rs11722228 in 300 individuals, and ABCG2rs2231142 and SLC22A12rs893006 in 297 individuals were successfully genotyped. The remaining three samples were not successfully genotyped due to volume insufficiency. Melting curves and DNA sequencing results are presented in Fig. 1. The 

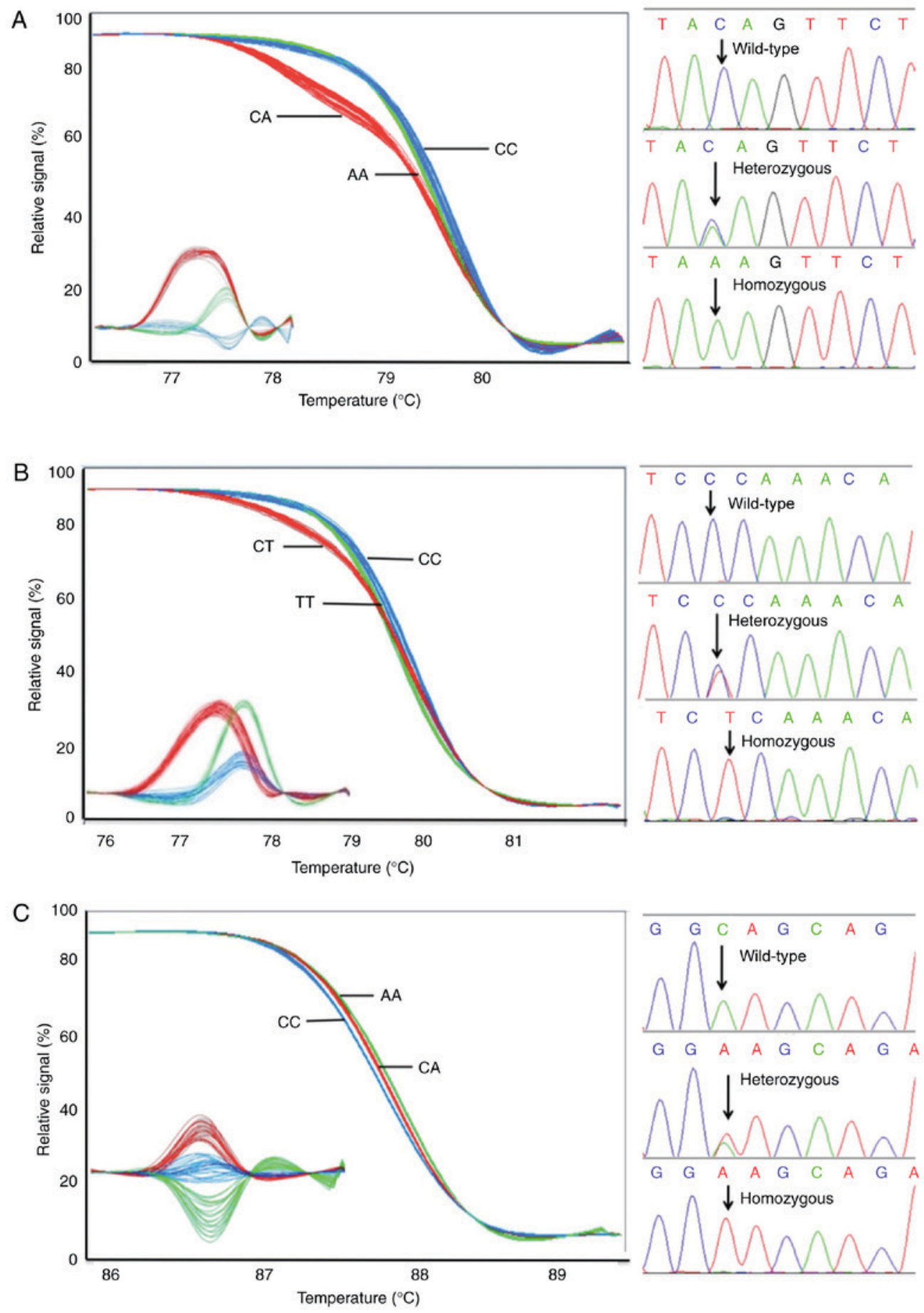

Figure 1. Melting curves and DNA sequencing. A Roche480 2.0 software analysis system presented two interfaces for analysis to demonstrate genotyping constituting the melting curves and temperature-shifted difference plots (left panel) of the single nucleotide polymorphisms: (A) ABCG2rs2231142, (B) SLC2A9rs11722228 and (C) SLC22A12rs893006. The sequencing results presented in the right panel of each melting curve were matched with results analyzed from high-resolution melting. ABCG2, ATP-binding cassette sub-family G member 2. SLC2A9, solute carrier family 2 member 9; SLC22A12, solute carrier family 22 member 12 .

reliability of the HRM assay was confirmed by the results obtained from DNA sequencing. The genotype frequencies of all three SNPs (SLC2A9rs11722228, SLC22A12rs893006 and ABCG2rs2231142) were in accordance with the Hardy-Weinberg equilibrium $(\mathrm{P}=0.73,0.17$ and 0.40 , respectively). The T allele of SLC2A9rs11722228 exhibited a frequency of $30.5 \%$, with genotype frequencies of $49.33,40.33$ and $10.33 \%$ for CC, CT and TT, respectively. The A allele of SLC22A12rs893006 had a frequency of $23.4 \%$, with genotype frequencies of $57.23,38.72$ and $4.05 \%$ for CC, CA and AA, respectively. Additionally, the A allele of ABCG2rs2231142 exhibited a frequency of $33.7 \%$, with genotype frequencies of $44.44,43.87$ and $11.78 \%$ for CC, CA and AA, respectively (Table III).

Sub-analysis of SUA levels by genotype. ANOVA was used to evaluate the differences in SUA levels within in each genotype. For SLC2A9rs11722228, the SUA concentration of the 
Table III. Allele and genotype frequency of SLC2A9rs11722228, SLC22A12rs893006 and ABCG2rs2231142 according to sex.

\begin{tabular}{|c|c|c|c|}
\hline Characteristic & Male & Female & Total \\
\hline Total no. & 170 & 130 & 300 \\
\hline Serum uric acid, $\mu \mathrm{mol} / 1$ & $461.8 \pm 91.4$ & $348.2 \pm 76.4$ & $412.6 \pm 102.1$ \\
\hline Age, years & $36.3 \pm 8.5$ & $35.7 \pm 8.8$ & $35.9 \pm 8.6$ \\
\hline \multicolumn{4}{|l|}{ SLC2A9rs11722228(\%) } \\
\hline $\mathrm{CC}$ & $78(45.88)$ & $70(53.85)$ & $148(49.33)$ \\
\hline $\mathrm{CT}$ & $74(43.53)$ & $47(36.15)$ & $121(40.33)$ \\
\hline $\mathrm{TT}$ & $18(10.59)$ & $13(10.00)$ & $31(10.33)$ \\
\hline \multicolumn{4}{|l|}{ Allele $(\%)$} \\
\hline $\mathrm{C}$ & $230(67.65)$ & $187(71.92)$ & $417(69.50)$ \\
\hline $\mathrm{T}$ & $110(32.35)$ & $73(28.08)$ & $183(30.50)$ \\
\hline \multicolumn{4}{|l|}{ SLC22A12rs893006 (\%) } \\
\hline $\mathrm{CC}$ & $95(55.88)$ & $75(59.06)$ & $170(57.23)$ \\
\hline CA & $67(39.41)$ & $48(37.80)$ & $115(38.72)$ \\
\hline AA & $8(4.71)$ & $4(3.41)$ & $12(4.05)$ \\
\hline \multicolumn{4}{|l|}{ Allele $(\%)$} \\
\hline $\mathrm{C}$ & $257(75.58)$ & $198(77.95)$ & $455(76.60)$ \\
\hline A & $83(24.42)$ & $56(22.05)$ & $139(23.40)$ \\
\hline \multicolumn{4}{|l|}{ ABCG2rs2231142(\%) } \\
\hline $\mathrm{CC}$ & $67(40.12)$ & $65(50.00)$ & $132(44.44)$ \\
\hline $\mathrm{CA}$ & $75(44.91)$ & $55(42.31)$ & $130(43.78)$ \\
\hline $\mathrm{AA}$ & 25 (14.97) & $10(7.69)$ & 35 (11.78) \\
\hline \multicolumn{4}{|l|}{ Allele (\%) } \\
\hline $\mathrm{C}$ & $209(62.57)$ & $185(71.15)$ & $394(66.33)$ \\
\hline A & $125(37.43)$ & $75(28.85)$ & $200(33.67)$ \\
\hline
\end{tabular}

Continuous data are presented as the mean \pm standard deviation. ABCG2, ATP-binding cassette sub-family G member 2. SLC2A9, solute carrier family 2 member 9; SLC22A12, solute carrier family 22 member 12 .

300 patients demonstrated no significant differences among the three genotype groups $(411.6 \mu \mathrm{mol} / 1$ for $\mathrm{CC}, 408.1 \mu \mathrm{mol} / 1$ for CT and $412.6 \mu \mathrm{mol} / 1$ for TT (Fig. 2A; P=0.424 vs. CC). For SLC22A12rs893006, subjects with AA and CA genotypes (319.2 \pm 80.7 and 409.6 $\pm 91.3 \mu \mathrm{mol} / 1$, respectively) exhibited lower SUA concentrations compared with those with the CC genotype $(417.1 \pm 110.8 \mu \mathrm{mol} / \mathrm{l})$; however, no significant difference was observed among the three genotypes (Fig. 2A; $\mathrm{P}=0.625$ vs. CC). Regarding ABCG2rs2231142, patients with the AA genotype had significantly higher concentrations of SUA $(435.7 \pm 106.39 \mu \mathrm{mol} / \mathrm{l})$ compared with those with CA or CC genotypes $(419.5 \pm 100.89$ and $398.0 \pm 100.84 \mu \mathrm{mol} / 1$, respectively; Fig. $2 \mathrm{~A}, \mathrm{P}=0.047$ vs. CC). Regarding SLC2A9 rs11722228, the SUA concentration among the three genotype groups of female patients did not demonstrate any significant differences $(359.1 \mu \mathrm{mol} / 1$ for CC, $311.5 \mu \mathrm{mol} / 1$ for CT and $381.2 \mu \mathrm{mol} / 1$ for TT; Fig. 2B, $\mathrm{P}=0.060$ vs. CC). For SLC22A12rs893006, the mean levels of SUA in female subjects were similar according to each genotypic group (347.5 $\mu \mathrm{mol} / 1$ for CC, $348.9 \mu \mathrm{mol} / 1$ for CA and $340.7 \mu \mathrm{mol} / 1$ for AA; Fig. $2 \mathrm{~B}, \mathrm{P}=0.979$ vs. CC). Regarding ABCG2rs2231142, the SUA concentration in female subjects did not demonstrate any significant differences among the three genotypic groups $(344.5 \mu \mathrm{mol} / 1$ for $\mathrm{CC}, 354.3 \mu \mathrm{mol} / 1$ for CA and $3388 \mu \mathrm{mol} / 1$ for AA; Fig. $2 \mathrm{~B}, \mathrm{P}=0.725$ vs. $\mathrm{CC}$ ). For SLC2A9rs11722228, the SUA concentration of male subjects demonstrated no significant differences among the three genotype groups (465.2 $\mu \mathrm{mol} / 1$ for $\mathrm{CC}, 455.3 \mu \mathrm{mol} / 1$ for $\mathrm{CT}$ and $473.2 \mu \mathrm{mol} / 1$ for TT; Fig. $3 \mathrm{~A}, \mathrm{P}=0.677$ vs. $\mathrm{CC}$ ). Regarding SLC22A12rs893006, male subjects with AA and CA genotypes ( $416.3 \mu \mathrm{mol} / 1$ and $452.9 \mu \mathrm{mol} / 1$, respectively) exhibited suppressed SUA concentrations compare with those with the CC genotype (471.8 $\mu \mathrm{mol} / \mathrm{l})$; however, no significant differences were observed among the three genotypes (Fig. 2C, $\mathrm{P}=0.154$ vs. CC). For ABCG2rs2231142, the SUA concentration exhibited by male subjects demonstrated no significant differences among the three genotypic groups (449.9 $\mu \mathrm{mol} / 1$ for $\mathrm{CC}, 467.3 \mu \mathrm{mol} / 1$ for $\mathrm{CA}$ and $474.5 \mu \mathrm{mol} / 1$ for AA; Fig. 2C, P=0.389 vs. CC).

Age-adjusted OR and 95\% CI of hyperuricemia for ABCG2rs2231142 genotypes. An age-adjusted logistic regression analysis was used to verify the association of ABCG2rs2231142 genotypes and hyperuricemia, as presented in Table IV. A total of 100 subjects were randomly selected from the hyperuricemia and normal SUA groups for this analysis. 

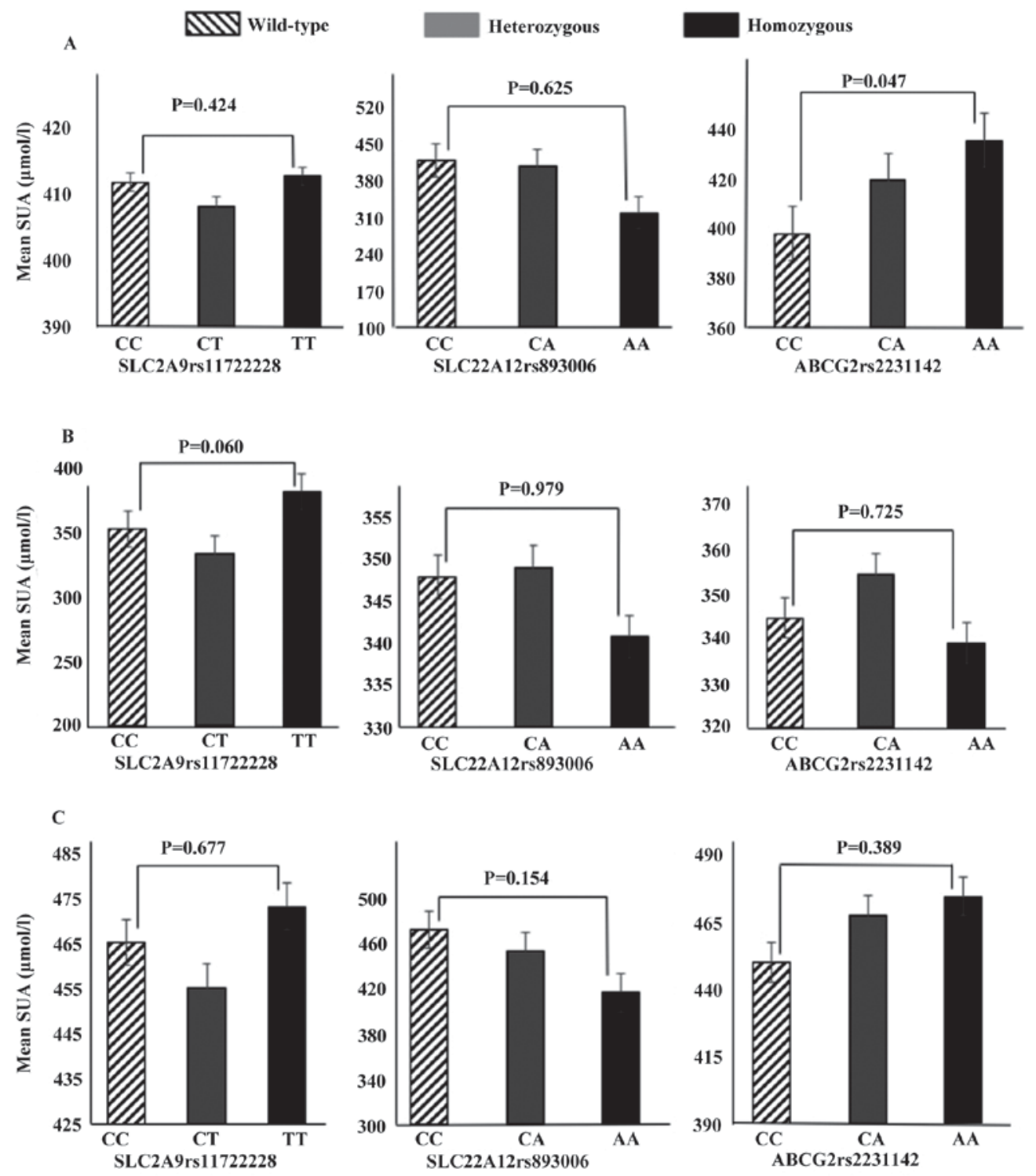

Figure 2. Mean of SUA among the three genotypes of single nucleotide polymorphisms (ABCG2rs2231142, SLC2A9rs11722228 and SLC22A12rs893006), according to sex. Analysis of SUA levels of wild-type, heterozygous and homozygous from (A) total, (B) female and (C) male data. Statistical differences between groups were determined by a one-way analysis of variance followed by the Least Significant Difference post-hoc test. SLC2A9, solute carrier family 2 member 9; SLC22A12, solute carrier family 22 member 12; SUA, serum uric acid.

The results revealed that the CA genotype frequency was $58 \%$ in the hyperuricemia group and $46 \%$ in the normal SUA group $(\mathrm{OR}=2.405 ; \mathrm{P}=0.036)$. The $\mathrm{AA}$ genotype frequency was $13 \%$ in the hyperuricemia group and $8 \%$ in the normal SUA group (OR=1.133; $\mathrm{P}=0.043)$. The A allele of $\mathrm{ABCG} 2 \mathrm{rs} 2231142$ exhibited a significant association with an increased risk of hyperuricemia.

Linkage disequilibrium analysis for SLC22A12rs893006 and SLC22A12rs1529909. The present study reported that the SLC22A2rs1529909 mutation (T/C, 146 bp) was always accompanied by the SLC22A12rs893006 mutation when SLC22A12rs893006 was genotyped via HRM curves. Additionally, computational analysis of linkage disequilibrium statistics was performed in the present study using Haploview 4.2 software (17). The results demonstrated that the variants rs893006 and rs1529909 of the SLC22A12 gene were in complete linkage disequilibrium: $\mathrm{R}^{2}>0.8$ (Fig. 3).

\section{Discussion}

In the present study, the prevalence of hyperuricemia was $32.6 \%$ (46.5\% in men and $27.7 \%$ in women) in the Chinese population of the southeast coastal region, which was notably higher compared with those reported in other regions, including $24.4 \%$ in Bangkok (59\% in men and $11 \%$ in women) (18), $21.4 \%$ in the United States (9) and $25.8 \%$ in Japan (5). Unexpectedly, the data of the present study revealed that the youngest group (20-30-years-old) exhibited higher mean SUA levels $(420.2 \pm 104.9 \mu \mathrm{mol} / \mathrm{l})$ compared with the other age groups (30-40 years old and 40-50 years old). This suggested that dietary habits, including high seafood in take, and lifestyle may partly contribute to high SUA levels; however, genetic factors may also serve an important role in affecting SUA levels. In addition, a high incidence of hyperuricemia within the younger ages groups in this region was observed in the present study. 
Table IV. Age-adjusted OR and 95\% CI of hyperuricemia for ABCG2rs2231142 genotypes.

\begin{tabular}{|c|c|c|c|c|c|c|c|c|c|}
\hline \multirow[b]{2}{*}{ Genotype } & \multicolumn{3}{|c|}{ Control } & \multicolumn{3}{|c|}{ Case } & \multirow[b]{2}{*}{ OR } & \multirow[b]{2}{*}{ P-value } & \multirow[b]{2}{*}{$95 \% \mathrm{CI}$} \\
\hline & Female & Male & Total & Female & Male & Total & & & \\
\hline $\mathrm{CC}$ & 14 & 32 & 46 & 8 & 21 & 29 & 1 & N/A & Reference \\
\hline CA & 15 & 31 & 46 & 20 & 38 & 58 & 2.05 & 0.036 & $0.93-4.49$ \\
\hline AA & 2 & 6 & 8 & 3 & 10 & 13 & 1.13 & 0.043 & $0.69-1.83$ \\
\hline
\end{tabular}

CI, confidence interval; OR, odds ratio; N/A, not applicable.

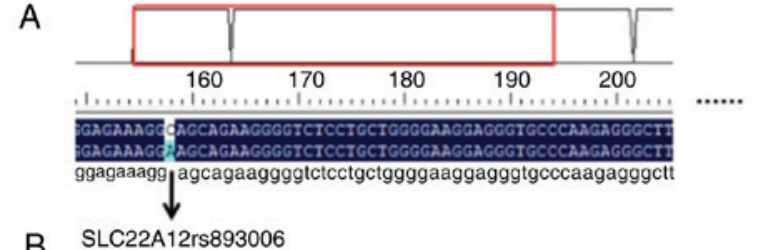

B

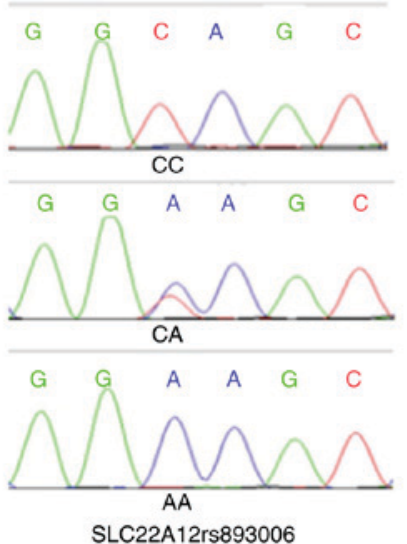

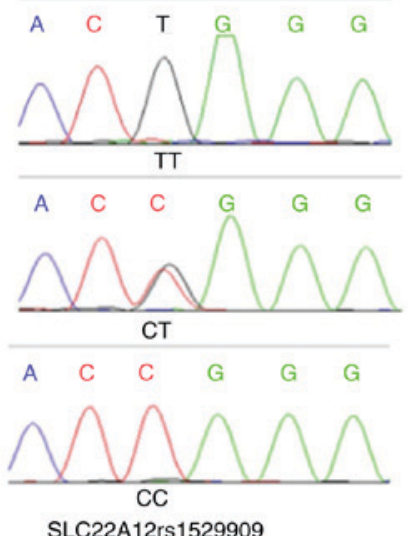

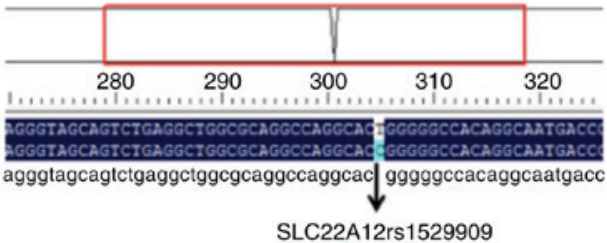

C

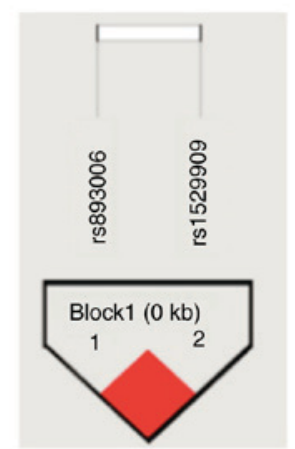

Figure 3. Haploview results for SLC22A12rs893006 and SLC22A12rs1529909. (A) Results of sequencing compared with the NCBI database via DNAMAN 8.0 software. (B) Sequencing results matched with those analyzed from high-resolution melting. (C) Linkage disequilibrium analysis via Haploview software (Haploview 4.2). SLC2A9, solute carrier family 2 member 9; SLC22A12, solute carrier family 22 member 12.

The ABCG2 gene encodes a membrane transporter belonging to the ATP-binding cassette superfamily of membrane transporters, which mediates urate excretion via the proximal renal tubule (19). A meta-analysis of 28,141 European individuals revealed that the gene exhibiting the strongest effects on SUA levels was SLC2A9, and the second was ABCG2 (7). Conversely, a GWAS on the Chinese population suggested that the effects of ABCG2 on SUA levels were stronger compared with SLC2A9 (20). In the present study, the variants of ABCG2rs2231142 were analyzed using PCR-HRM; SUA levels were compared among the three genotype groups. The results of the present study demonstrated that the genotype frequencies of CC, CA and AA were $44.44,43.87$ and $11.78 \%$, respectively, in this region. Furthermore, the present study reported that individuals harboring the AA genotype of ABCG2rs2231142 exhibited significantly higher concentrations of SUA compared with the CA or CC genotypes. It has been reported that different genotypes may affect SUA levels according to sex $(12,21)$. Some variation between the male and female data was demonstrated in the results of the present study; however, such differences were not revealed to be statistically significant. This suggested that the genotype of homozygous mutation may have a closer association with high concentrations of SUA levels compared with the heterozygous and wild-type. ABCG2rs2231142 is prevalent in the majority of populations, with the A allele frequency varying between $1 \%$ in African and 29\% in Southeast Asian populations (12). The frequency of the A allele in the present study was $33.67 \%$, which was similar to other East Asian regions (40.5\% in Taiwan and $31.0 \%$ in Japan) $(21,22)$. The age-adjusted logistic regression analysis between the hyperuricemia and normal groups demonstrated that the A allele may increase susceptibility to hyperuricemia $(\mathrm{OR}=2.405,1.133$ for $\mathrm{CA}$ and AA, respectively). Therefore, it can be suggested that the ABCG2rs2231142 mutation may represent an important factor contributing to the high incidence of hyperuricemia in the region investigated by the present study.

SLC2A9 is located in chromosome 4 and encodes GLUT9, which is responsible for the reabsorption of UA and the exchange of glucose and fructose in the basolateral membranes of renal proximal tubules $(23,24)$. SLC22A12, encoding URAT1, is a member of the organic anion transporter family and primarily regulates the renal tubular reabsorption of UA (10). The present study reported that individuals carrying wild-type alleles in SLC2A9rs11722228 and 
SLC22A12rs893006 exhibited higher SUA levels compared with those possessing variant alleles; however, the differences did not reach statistical significance. A larger difference in the mean SUA levels between SLC22A12rs893006 genotypes was detected in males compared with females. Previous studies investigating the American Indian, Japanese and Korean populations have identified variants of SLC2A9 to be principal determinants of SUA levels $(22,25,26)$. In particular, a meta-analysis of European populations demonstrated that the SLC2A9rs734553 polymorphism may be an independent genetic marker associated with SUA levels (7). It has been reported that Japanese population revealed the polymorphism SLC22A12rs893006 to be an independent genetic marker for predicting hyperuricemia (11). Although no significant differences in SUA levels were observed among the genotype groups in the present study, non-significant associations between lower SUA levels with particular genetic variants, such as the A allele in SLC22A12rs893006, were revealed, which was in accordance with the findings of a previous study (9). Minor differences in the data of the present study may be due to differing effects of genetic variants exerted on SUA levels, which may be attributed to various ethnic groups, diet lifestyle and any potential underlying health conditions.

Notably, the present study revealed that the variants rs893006 and rs1529909 of the SLC22A12 gene were in complete linkage disequilibrium; to the best of our knowledge, this has not been reported in previous GWAS. The results of the present study suggested that these two linked loci may possibly encode a particular structure in URAT1 affecting the function of the UA transporter. Further functional investigation is required to confirm the results of the present study and improve understanding of the underlying biological mechanisms of the effects exerted by these polymorphism on URAT1.

However, there were certain limitations to the present study. Firstly, all SNPs that have been confirmed to affect SUA levels were not evaluated in other ethnic groups. Additionally, the present study aimed to investigate the effects of gene mutations on SUA levels; however, lifestyle factors and other parameters of metabolic conditions were not considered. Therefore, further investigation into other functional SNPs and an analysis of the numerous contributing factors in the Chinese population of the southeast coastal region may be conducted in the future.

In conclusion, the present study reported a high incidence rate of hyperuricemia in the Chinese population of the southeast coastal region, which was significantly associated with variants in ABCG2rs2231142; however, further investigation into whether SLC2A9rs11722228 and SLC22A12rs893006 may act as important genetic candidates in the regulation of SUA levels in this region is required.

\section{Acknowledgements}

Not applicable.

\section{Funding}

The present study was supported by the Department of Education, Guangdong Government Scheme for Research and Control of Infectious Diseases.

\section{Availability of data and materials}

The datasets used and/or analyzed during the current study are available from the corresponding author on reasonable request.

\section{Authors' contributions}

QZ made substantial contributions to the design of the present study, data acquisition, data analysis and reviewed the manuscript. XY made substantial contributions to the design of the present study and contributed to the writing of the manuscript. YX conducted the high-resolution melting methods and analyzed the statistical data. KL collected clinical characteristics of the subjects enrolled in the present study and revised the manuscript for important intellectual content. $\mathrm{XJ}$ revised the manuscript for important intellectual content and participated in the design of questionnaire survey. $\mathrm{XL}$ and $\mathrm{YW}$ drafted the manuscript and participated in all experiments of the present study. All authors read and approved the final manuscript.

\section{Ethics approval and consent to participate}

The study protocol was approved by the Ethics Committee of The First Affiliated Hospital of Shantou University Medical College. All participants provided written informed consent.

\section{Patient consent for publication}

All participants gave written informed consent including genotyping, and informed consent for the publication of any associated data and accompanying images.

\section{Competing interests}

The authors declare that they have no competing interests.

\section{References}

1. Bobulescu IA and Moe OW: Renal transport of uric acid: Evolving concepts and uncertainties. Adv Chronic Kidney Dis 19: 358-371, 2012.

2. Puddu P, Puddu GM, Cravero E, Vizioli L and Muscari A: The relationships among hyperuricemia, endothelial dysfunction and cardiovascular diseases: Molecular mechanisms and clinical implications. J Cardiol 59: 235-242, 2012.

3. Reginato AM, Mount DB, Yang I and Choi HK: The genetics of hyperuricaemia and gout. Nat Rev Rheumatol 8: 610-621, 2012.

4. Sakurai H: Urate transporters in the genomic era. Curr Opin Nephrol Hypertens 22: 545-550, 2013.

5. Nath SD, Voruganti VS, Arar NH, Thameem F, Lopez-Alvarenga JC, Bauer R, Blangero J, MacCluer JW, Comuzzie AG and Abboud HE: Genome scan for determinants of serum uric acid variability. J Am Soc Nephrol 18: 3156-3163, 2007.

6. Yang Q, Guo CY, Cupples LA, Levy D, Wilson PW and Fox CS: Genome-wide search for genes affecting serum uric acid levels: The framingham heart study. Metabolism: 54: 1435-1441, 2005.

7. Kolz M, Johnson T, Sanna S, Teumer A, Vitart V, Perola M, Mangino M, Albrecht E, Wallace C, Farrall M, et al: Meta-analysis of 28,141 individuals identifies common variants within five new loci that influence uric acid concentrations. PLoS Genet 5: e1000504, 2009.

8. Dehghan A, Köttgen A, Yang Q, Hwang SJ, Kao WL, Rivadeneira F, Boerwinkle E, Levy D, Hofman A, Astor BC, et al: Association of three genetic loci with uric acid concentration and risk of gout: A genome-wide association study. Lancet 372: 1953-1961, 2008 
9. Ware EB, Riehle E, Smith JA, Zhao W, Turner ST, Kardia SL and Lieske JC: SLC2A9 genotype is associated with SLC2A9 gene expression and urinary uric acid concentration. PLoS One 10: e0128593, 2015.

10. Cho SK, Kim S, Chung JY and Jee SH: Discovery of URAT1 SNPs and association between serum uric acid levels and URAT1. BMJ Open 5: e009360, 2015.

11. Shima Y, Teruya $\mathrm{K}$ and Ohta $\mathrm{H}$ : Association between intronic SNP in urate-anion exchanger gene, SLC22A12 and serum uric acid levels in Japanese. Life Sci 79: 2234-2237, 2006.

12. Yang B, Mo Z, Wu C, Yang H, Yang X, He Y, Gui L, Zhou L, Guo H, Zhang X, et al: A genome-wide association study identifies common variants influencing serum uric acid concentrations in a Chinese population. BMC Med Genomics 7: 10, 2014.

13. Guohong Z, Min S, Duenmei W, Songnian H, Min L, Jinsong L, Hongbin L, Feng Z, Dongping T, Heling Y, et al: Genetic heterogeneity of oesophageal cancer in high-incidence areas of southern and northern China. PLoS One 5: e9668, 2010.

14. Zheng X, Lin M, Yang H, Pan MC, Cai YM, Wu JR, Lin F, Zhan XF, Luo ZY, Yang HT and Yang LY: Molecular epidemiological characterization and health burden of thalassemias in the Chaoshan region, People's Republic of China. Hemoglobin 40 138-142, 2016

15. Huang H, Su M, Li X, Li H, Tian D, Gao Y and Guo Y: Y-chromosome evidence for common ancestry of three Chinese populations with a high risk of esophageal cancer. PLoS One 5: e11118, 2010

16. Iacumin L, Ginaldi F, Manzano M, Anastasi V, Reale A, Zotta T, Rossi F, Coppola R and Comi G: High resolution melting analysis (HRM. as a new tool for the identification of species belonging to the Lactobacillus casei group and comparison with species-specific PCRs and multiplex PCR. Food Microbiol 46 357-367, 2015.

17. Barrett JC, Fry B, Maller J and Daly MJ: Haploview: Analysis and visualization of LD and haplotype maps. Bioinformatics 21 : 263-265, 2005
18. Li J, Dong BR, Lin P, Zhang J and Liu GJ: Association of cognitive function with serum uric acid level among Chinese nonagenarians and centenarians. Exp Gerontol 45: 331-335, 2010

19. Lima A, Azevedo AR, Sousa H, Bernardes M, Medeiros R and Seabra V: AB0223 SLC19A1, SLCO1B1 and ABCG2 polymorphisms are associated with methotrexate-related gastrointestinal toxicity in Portuguese rheumatoid arthritis patients. Ann Rheum Dis 73 (Suppl 2): S877-S877, 2014

20. Ishikawa T, Aw W and Kaneko K: Metabolic interactions of purine derivatives with human ABC transporter ABCG2: Genetic testing to assess gout risk. Pharmaceuticals (Basel) 6: 1347-1360, 2013.

21. Cheng ST, Wu S, Su CW, Teng MS, Hsu LA and Ko YL: Association of ABCG2 rs2231142-A allele and serum uric acid levels in male and obese individuals in a Han Taiwanese population. J Formos Med Assoc 116: 18-23, 2017.

22. Hamajima N, Okada R, Kawai S, Hishida A, Morita E, Yin G, Wakai K, Matsuo H, Inoue H, Takada Y, et al: Significant association of serum uric acid levels with SLC2A9 rs11722228 among a Japanese population. Mol Genet Metab 103: 378-382, 2011.

23. Yamagishi K, Tanigawa T, Kitamura A, Köttgen A, Folsom AR and Iso H; CIRCS Investigators: The rs2231142 variant of the ABCG2 gene is associated with uric acid levels and gout among Japanese people. Rheumatology (Oxford) 49: 1461-1465, 2010.

24. Das Gupta E, Sakthiswary R, Lee SL, Wong SF, Hussein H and Gun SC: Clinical significance of SLC2A9/GLUT9 rs11722228 polymorphisms in gout. Int J Rheum Dis 21: 705-709, 2018.

25. Voruganti VS, Franceschini N, Haack K, Laston S, MacCluer JW, Umans JG, Comuzzie AG, North KE and Cole SA: Replication of the effect of SLC2A9 genetic variation on serum uric acid levels in American Indians. Eur J Hum Genet 22: 938-943, 2014.

26. Kim YS, Kim Y, Park G, Kim SK, Choe JY, Park BL and Kim HS: Genetic analysis of ABCG2 and SLC2A9 gene polymorphisms in gouty arthritis in a Korean population. Korean J Intern Med 30: 913-920, 2015. 